is multiple sclerosis. ${ }^{89}$ Several groups expressed some optimism in reporting the results of treating this disease with cytotoxic drugs in combination with A.L.G. The uncontrolled nature of these studies and doubts about attributing multiple sclerosis to immunological causes left orthodox neurological opinion deeply sceptical about their findings. Similar claims have been made for A.L.G. treatment in other putative autoimmune diseases. ${ }^{1011}$ The results of treating a few patients with dermatomyositis, systemic lupus erythematosus, and autoimmune disorders affecting the bone marrow with A.L.G. were described by other speakers. To an even greater extent their findings are difficult to assess, since different batches of A.L.G. with diverse biological properties have been given with variable schedules of other drugs to small numbers of patients with diseases which run an unpredictable course. These shortcomings were freely admitted and reflect the associated problems of ethics and organisation which need to be overcome. Nevertheless, these pilot studies have been sufficiently encouraging to justify setting up double-blind controlled trials, without which the therapeutic value of A.L.G. in the treatment of such diseases is unlikely to be established. The theoretical importance of such studies is beyond question, since an unequivocally beneficial response to immunosuppressive treatment in a disease such as multiple sclerosis would favour the still contentious view that this disease has an immunological basis.

The conference did not settle any of the already wellrecognized problems about the use of A.L.G. in clinical practice. It simply reiterated the need for carefully controlled studies in patients with severe, life-threatening autoimmune disorders. The preparations of A.L.G. now available are still lamentably variable in their biological properties but they have at least reached a degree of refinement which has removed many of their worst dangers. Nevertheless, the risks of profound immunosuppression which already existed with other agents are made worse by the addition of A.L.G. to treatment schedules; there were several indications at the conference that overall suppression of bone-marrow function and cytomegalo-virus infections may be particular hazards of such therapy. Certainly A.L.G. treatment will demand careful monitoring, and the need for devising more selective methods of controlling unwanted immune reactions remains urgent.

\footnotetext{
1 James, K., Clinical and Experimental Immunology, 1967, 2, 615.

2 Lance, E. M., Clinical and Experimental Immunology, 1960, 6, 789.

3 Floersheim, G. L., Lancet, 1969, 1, 228.

4 Turk, J. L., and Willoughby, D. A., Antibiotics and Chemotherapy (Basel), 1969, 15, 267 .

5 Denman, A. M., Clinical and Experimental Immunology, 1969, 5, 217.

6 Proceedings of the Symposium on Antilymphocyte Globulin in Clinical Practice, ed. R. Roussell, in press.

Hoover, R., and Fraumeni, J. F., Lancet, 1973, 1, 55.

8 Ring, J., et al., Lancet, 1974, 2, 1093.

9 Lance, E. M., Knight, S. C., and Abbosh, J., Clinical and Experimental Immunology, in press.

10 Trepel, F., et al., Klinische Wochenschrift, 1968, 46, 856.

11 Pirofsky, B., et al., Fournal of the American Medical Association, 1969, 210, 1059.
}

\section{Steroids in the Eye}

Ophthalmology tends to receive an ever-decreasing place in the undergraduate medical curriculum, but one topic still universally taught is the diagnosis and treatment of the acute red eye. This is such a common problem that it would be quite impossible for all cases to be seen by trained ophthalmologists, and yet on some occasions the correct diagnosis cannot be made without either specialist knowledge or equipment.
Judgement of which cases to refer to hospital and when can present difficulties for the general practitioner.

Corneal foreign bodies are a frequent cause of red eye, when the history and sudden onset usually give a clear indication of the cause and the offending speck of dirt can usually be seen easily with the aid of a pencil torch. The treatment is simply the removal of the foreign body and patching the eye until the epithelium has regrown. Apart from trauma, the common causes of a red eye are acute closed-angle glaucoma, uveitis, keratitis, episcleritis and scleritis, and conjunctivitis. In some of these conditions the role of local steroids is all important: they may be either a major therapeutic weapon or a cause of serious complications.

Acute closed-angle glaucoma is often less painful than is commonly supposed, but there is the invaluable physical sign that the pupil will not respond to light owing to ischaemia of the iris. It is usually half dilated and may be oval with the long axis lying vertically. There is no other cause for this pupil reaction; the patient should receive carbonic anhydrase inhibitors and miotics and be referred to hospital at once.

Uveitis is one condition in which the main treatment is the use of local steroids: though these do not cure but merely control the inflammatory damage inside the eye. A delay in treatment can lead to extensive iris adhesions (synechiae), either to the lens or to the angle of the anterior chamber, both of which may result in secondary glaucoma. A patient with a known history of uveitis who presents with a red eye should be assumed to have had a relapse and treated with steroids again.

Keratitis represents a more difficult diagnosis. Marginal corneal ulceration ${ }^{1}$ responds specifically to local steroids. Most of the other causes of keratitis are viral, and by far the most important is the recurring keratitis caused by herpes simplex virus. This condition tends to affect one eye repeatedly and is very often associated with other stigmata of a disease such as cold sores round the face and mouth. Classically, herpes simplex gives rise to dendritic ulcers, which if large enough may be recognized with the aid of fluorescein eye drops and a torch and magnifying glass. If local steroids are used in this condition the patient obtains subjective relief and the eye will become whiter but the ulcer will spread in both area and depth and corneal perforation may result. Local steroids are absolutely contraindicated in any patient with a history of herpes or in whom this diagnosis is suspected.

Scleritis is an uncommon disease in which the eye is locally red and exquisitely painful. The usual causes are rheumatoid arthritis and gout. Though steroids may be of benefit this is a serious eye condition which needs hospital supervision. Episcleritis is an inflammation of those tissues lying between the conjunctiva and sclera. It produces a mild discomfort, no discharge, and localized redness. The cause is often obscure, and the disease frequently recurs. Local steroids are of benefit but the diagnosis is difficult without the magnification of a slit lamp.

Perhaps the greatest source of confusion comes in the diagnosis of conjunctivitis. Conjunctivitis is invariably accompanied by a discharge from the eye, the vision is not affected, and the pupil reacts quite normally. If the discharge is obviously purulent a local antibiotic should be applied at frequent intervals for several days. This will usually resolve the matter, and steroids should not be used. If, however, the condition persists for longer than this the possibility of a virus conjunctivitis should be considered. Most of these have a natural history lasting a week or two and will resolve regardless of any treatment. Though local steroids are useful in some instances their use requires expert knowledge. Another 
major cause of confusion in the diagnosis of conjunctivitis is the presence of dry eyes, which can occur in such conditions as rheumatoid arthritis, or after prolonged staphylococcal blepharitis. These patients of ten present with gritty and uncomfortable eyes and the condition may be easily mistaken for conjunctivitis. A local antibiotic may then be used and found ineffective; if local steroids, either alone or combined with antibiotics, are then given they will give some symptomatic relief simply because they provide fluid over the eye. These patients are specially at risk if placed on long-term steroids. About $10 \%$ of the population react to local steroids with a high increase of intraocular pressure, and serious damage may result from secondary glaucoma from a treatment which is both unnecessary and ineffective.

Local steroids should, then, be used only in known cases of uveitis and in those patients in whom the diagnosis of marginal corneal ulceration is certain. If they are prescribed they should not be continued for more than two or three weeks without specialist advice and should never be given when the diagnosis is in question or in patients with a history of herpes simplex infection.

${ }^{1}$ British Medical fournal, 1971, 1, 303.

\section{Coronary Costs}

National affluence, though in many ways desirable, has ill effects: one of these is wastefulness in medical care. Though N.H.S. staff did not do well in the 1960 s a generally satisfactory economy allowed moderate expansion of the Service's resources, much of which was directed to hospital care. Sudden adverse economic influences and unparalleled inflation have squeezed the N.H.S. and it appears that standards can be maintained only if economy and efficiency can be pursued-and if affluence has made the N.H.S. inefficient, poverty may yet create the environment in which cost effectiveness becomes something more than a talking point at symposia. Information is badly needed, especially in diseases such as myocardial infarction which are such expensive consumers of resources.

A recent study from the U.S.A. ${ }^{1}$ looked at a hospital's case records of myocardial infarction in the years 1939, 1949, 1959, and 1969. Data collected included age, sex, and clinical outcome, details of nursing observations, use of oxygen, analgesia, sedation, the frequency of diagnostic procedures, and an assessment of the severity of the episodes. The authors, making the null hypothesis that mortality in each severity group was unchanged from 1939 onwards, found an excess of deaths only in the first year studied. The hospital did not have a coronary care unit, but the acceptance of these results means that all the many other "advances" in management of myocardial infarction had been without benefit in terms of hospital survival. Possibly improvements have been obscured by a bias against survival from the modern trend to quicker admission and by some defects in the retrospective assessment of severity owing to the data necessarily being incomplete.

The other data are legitimate and thought provoking. There was a linear rise in numbers of E.C.G.s, $x$-rays, and bacteriological tests, an exponential increase in chemical investigations, and an increase in sedation and the use of oxygen. There was no appreciable change in the time spent in hospital in survivors. Nursing recordings were maintained for a shorter period in 1949 and 1959, but this trend was reversed by 1969.
The amount of investigation and clinical activity did not correlate with the assessment of severity-an indication that routine rather than need dictated much of what was done. This is doubtless the case in many units for many E.C.G. requests, radiological and biochemical procedures, and for four-hourly nursing recordings after the third day in patients with mild episodes. Simply because the severity assessments were questionable, it is not possible to conclude that these various procedures did not influence survival; and survival is not the only important issue. Minimum disturbance during the initial illness is a worthy objective, and one which may well depend on observation and investigation; and the quality and length of survival after discharge are more relevant to society than the cost-effectiveness of short-term hospital survival. Most physicians will feel reasonably sure that basic investigative data, though they might not influence immediate survival, do have a lot to do with decision-taking that may influence longterm health.

The duration of stay in this American study was influenced by neither severity nor the passage of time. Even in 1969 survivors were averaging almost a month in hospital. That the mildly ill patients served this long sentence suggests a very conservative, old fashioned approach, or again raises doubts about the severity data. Though it is still impossible to resolve the argument about home versus hospital care there is at least cast-iron evidence that early mobilization of patients with uncomplicated infarcts is not harmful as measured by subsequent angina pectoris, ventricular aneurysm formation, cardiac failure, or long-term survival. ${ }^{2}$ Any disadvantage in healing because of more rapid mobilization is fully compensated by a presumed reduction in venous thromboembolism, muscle wasting, and the resultant decline in exercise tolerance. Furthermore early discharge presumably brings benefits in psychological terms, in both the short and long term.

These studies should stimulate more economic use of hospital beds and facilities. Whatever the arguments about initial coronary care, apparently the later aspects canwithout clinical disadvantage-be moved away from the hospital sector. Any move in that direction would require co-operation between family doctors and hospital doctors and perhaps some strengthening of the home nursing services. Savings made might be diverted towards initial coronary care, where immediate access to repeated defibrillation remains the only way of reducing deaths from cardiac arrest. ${ }^{3}$ Almost certainly, a system of very rapid admission and detailed supervision for three days would cover almost all arrests and less serious but equally treatable complications.

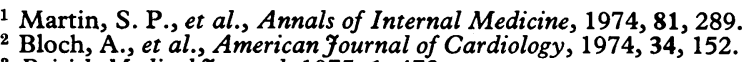

3 British Medical fournal, 1975, 1, 473 .

\section{Side Effects of Methyldopa}

Screening surveys have shown that $15-30 \%$ of adults have raised blood pressure levels ${ }^{1}$; most are unaware of the presence or significance of their hypertension. When treatment is necessary the severity and nature of the underlying disease process influence the choice of drug.

Methyldopa has been widely prescribed since $1960,{ }^{2}$ but its side effects have proved less acceptable in recent years in patients with mild or symptomless hypertension. Johnson et $a .^{3}$ found side effects in $72 \%$ of patients, including weight 